\title{
THE CHARACTERISATION OF SCORING FUNCTIONS
}

\author{
MICHAEL A. B. DEAKIN
}

(Received 2 June 1999; revised 7 December 2000)

Communicated by V. Stefanov

\begin{abstract}
The literature on subjective probabilities contains a number of functions that have been proposed as 'scoring functions'. The principal requirement is that, with several events that may occur in the future and to which subjective 'probabilities' are assigned, the expected score given by these 'probabilities' will be extremised if the values assigned equal the 'true probabilities' of the various outcomes. This article discusses the question of what other scoring functions might be used (beyond those so far proposed).
\end{abstract}

2000 Mathematics subject classification: primary $60 \mathrm{~A} 99$.

\section{The situation}

The topic of 'scoring functions' has developed quite a large literature. See, for example, discussions by Winkler [7-9], by Savage [5] and by Schervish [6]. For a discussion of the underlying principles and of the proposed applications of the theory, see also de Finetti [3, 185-198].

These analyses all consider a 'multi-event', that is to say a set of $n$ mutually incompatible events, one and only one of which will actually occur (at some time in the future). The details that follow will be presented in terms of the particular case $n=3$. The reader will easily see however that this case is typical and that the results readily generalise. The case $n=3$ is adopted here both for definiteness and for notational convenience.

In fact, it is the difference between the cases $n=2, n>2$ that is the most interesting, as it raises points of principle that are resolved already when we progress beyond the case $n=3$. The case $n=2$ formed the subject of an earlier paper by the

(C) 2001 Australian Mathematical Society 0263-6115/2001 \$A2.00+0.00 
author [2]. The discussion presented here proceeds in terms similar (modulo some comments to be made below) to those used there in that special case, but the notation is different.

Suppose that a tipster (possibly even an expert in whatever the relevant field might be) assigns, in advance, positive numbers $P, Q, R$ to each of the three possible outcomes $E_{1}, E_{2}, E_{3}$ of some happening ('multi-event'). Suppose also that the best possible such estimates are the probabilities $p, r, q$ of these events actually occurring.

The scoring function will be a real-valued function $f(P, Q, R ; P)$ whose value is awarded to the tipster in the event that $E_{1}$ occurs; similarly the score will be $f(P, Q, R ; Q)$ if $E_{2}$ occurs and $f(P, Q, R ; R)$ if $E_{3}$ occurs.

The expected score $e(P, Q, R)$ will thus be

$$
e(P, Q, R)=p f(P, Q, R ; P)+q f(P, Q, R ; Q)+r f(P, Q, R ; R)
$$

and the scoring function is to be chosen in such a way that $e(P, Q, R)$ is extremised for the choice $(P, Q, R)=(p, q, r)$.

The object of this paper is to categorise the possible scoring functions $f(P, Q, R ; P)$ under this criterion as a necessary condition. Solutions to this problem have been given before using different methods and employing different criteria as to what constitutes an acceptable scoring function. Here, instead of quoting general properties of such functions, explicit formulae will be displayed, and it will be supposed throughout that $f(P, Q, R ; P)$ is symmetric in its first three variables.

Three scoring functions have wide currency; these are (see, for example, $[7,8]$ for these functions in the case $n=2$ ):

(2) The logarithmic function $f(P, Q, R ; P)=\ln P$.

(3) The quadratic function $f(P, Q, R ; P)=(P-1)^{2}+Q^{2}+R^{2}$.

(4) The so-called 'spherical function' $f(P, Q, R ; P)=P / \sqrt{P^{2}+Q^{2}+R^{2}}$.

The reader will note that it is not imposed at this stage that $P+Q+R=1$. It is however assumed already in the setting up of (1) that $p+q+r=1$. However at certain points in the argument that follows, it will be convenient not to use this information immediately.

[In his analysis, de Finetti goes further and shows that in the case of the quadratic function (3), a tipster adopting a strategy $P+Q+R \neq 1$ could always do better by replacing $P, Q, R$ by $P^{*}, Q^{*}, R^{*}$ respectively, where

$$
P^{*}=\frac{1+2 P-Q-R}{3}
$$

etc., and where clearly $P^{*}+Q^{*}+R^{*}=1$. This provides a strong incentive for the tipster to use probabilities for $P, Q, R$. The case of the logarithmic function, however, 
is different and here the requirement $P+Q+R=1$ needs to be imposed. The same is true of the 'spherical function'.]

It will be further noted that any combination $a+b f(P, Q, R ; P)$, where $a$ and $b$ are constants $(b \neq 0)$, may replace $f(P, Q, R ; P)$ without any alteration to the thrust of the argument. In the earlier study [2], $a, b$ were chosen to meet certain normalisation conditions. These will not be imposed in the present account.

Finally, $f_{1}(P, Q, R ; P)$ and $f_{2}(P, Q, R ; P)$ are both scoring functions then so is $a f_{1}(P, Q, R ; P)+b f_{2}(P, Q, R ; P)$, where once again $a$ and $b$ are constants (not both zero), certainly as long as $f_{1}, f_{2}$ are both maximised or both minimised at $(p, q, r)$, but in fact rather more generally.

\section{Symmetric functions}

It will be convenient to write the scoring functions in a somewhat different form to take account of the imposed symmetries. The theory is standard, see for example $[1,420-427]$. In the case of three variables $P, Q, R$, it is usual to express such symmetric functions in terms of three basis functions $P+Q+R, P Q+Q R+R P$ and $P Q R$ and this is typical of all cases. That any integral symmetric function of the $P, Q$ and $R$ may be expressed in terms of these functions is Newton's theorem [1, 438-444] and indeed many other (non-integral) symmetric functions may also be so expressed. This paper will use not Newton's theorem directly, but rather some elementary consequences of it.

Write $S_{1}=P+Q+R, S_{2}=P^{2}+Q^{2}+R^{2}, S_{3}=P^{3}+Q^{3}+R^{3}$. Then as the usual basic functions can be expressed in terms of these new ones any integral symmetric function of the $P, Q$ and $R$ may be expressed in terms of $S_{1}, S_{2}$ and $S_{3}$; again the result applies much more widely. These results readily generalise to any number of independent variables. Although they are not given explicitly, they are implicit in the discussion and the displayed equations in (for example) Chrystal's classic text [1, 437] and doubtless elsewhere.

Thus write

$$
f(P, Q, R ; P)=f\left(S_{1}, S_{2}, S_{3} ; P\right), \quad \text { etc. }
$$

\section{The basic condition}

We may now write $e(P, Q, R)$ in this notation and via the use of Lagrange multipliers seek to extremise

$$
\begin{aligned}
e^{*}= & p f\left(S_{1}, S_{2}, S_{3} ; P\right)+q f\left(S_{1}, S_{2}, S_{3} ; Q\right)+r f\left(S_{1}, S_{2}, S_{3} ; R\right) \\
& +\lambda_{1}\left(S_{1}-P-Q-R\right)+\lambda_{2}\left(S_{2}-P^{2}-Q^{2}-R^{2}\right)+\lambda_{3}\left(S_{3}-P^{3}-Q^{3}-R^{3}\right) .
\end{aligned}
$$


The relevant equations then become (with $S_{1}, S_{2}, S_{3}, P, Q, R$ all now being treated as independent variables)

$$
\begin{aligned}
& p \frac{\partial f\left(S_{1}, S_{2}, S_{3} ; P\right)}{\partial P}-\lambda_{1}-2 P \lambda_{2}-3 P^{2} \lambda_{3}=0, \\
& q \frac{\partial f\left(S_{1}, S_{2}, S_{3} ; Q\right)}{\partial Q}-\lambda_{1}-2 Q \lambda_{2}-3 Q^{2} \lambda_{3}=0, \\
& r \frac{\partial f\left(S_{1}, S_{2}, S_{3} ; R\right)}{\partial R}-\lambda_{1}-2 R \lambda_{2}-3 R^{2} \lambda_{3}=0, \\
& \frac{\partial}{\partial S_{1}}\left[p f\left(S_{1}, S_{2}, S_{3} ; P\right)+q f\left(S_{1}, S_{2}, S_{3} ; Q\right)+r f\left(S_{1}, S_{2}, S_{3} ; R\right)\right]+\lambda_{1}=0, \\
& \frac{\partial}{\partial S_{2}}\left[p f\left(S_{1}, S_{2}, S_{3} ; P\right)+q f\left(S_{1}, S_{2}, S_{3} ; Q\right)+r f\left(S_{1}, S_{2}, S_{3} ; R\right)\right]+\lambda_{2}=0, \\
& \frac{\partial}{\partial S_{3}}\left[p f\left(S_{1}, S_{2}, S_{3} ; P\right)+q f\left(S_{1}, S_{2}, S_{3} ; Q\right)+r f\left(S_{1}, S_{2}, S_{3} ; R\right)\right]+\lambda_{3}=0 .
\end{aligned}
$$

These equations are to be identities when $P=p, Q=q, R=r$ and thus from the first of them

$$
\frac{\partial f}{\partial p}=\frac{\lambda_{1}}{p}+2 \lambda_{2}+3 p \lambda_{3},
$$

while from the final three of the set, we may replace the $\lambda_{i}$ by functions of the $S_{i}$.

The result is to give an $f$ that (to revert to the $P$ in place of $p$ ) is of the form

$$
\begin{aligned}
f\left(S_{1}, S_{2}, S_{3} ; P\right)= & S\left(S_{1}, S_{2}, S_{3}\right) \ln P+A\left(S_{1}, S_{2}, S_{3}\right) \\
& +P B\left(S_{1}, S_{2}, S_{3}\right)+P^{2} C\left(S_{1}, S_{2}, S_{3}\right),
\end{aligned}
$$

where $S=\lambda_{1}, B=2 \lambda_{2}, C=3 \lambda_{3} / 2$ and $A$ is a constant of integration.

The result is a sum of a logarithmic function (to be briefly considered below) and a function quadratic in $P$. Because the scoring function can be considered as a sum of the independent contributions of its various components these two functions can be analysed independently of one another. The logarithmic scoring function will now be discussed. The other component will be considered later.

\section{The logarithmic scoring function}

The theory of the logarithmic scoring function may be summarised briefly. If $f(P, Q, R ; P)$ is in fact independent of $Q, R$, then the condition $P+Q+R=1$ must be imposed, in which case we find $f(P, Q, R ; P)=\ln P$. For a discussion, see for example Winkler [9]. 
More generally however, from (6), consider the possibility of a more general logarithmic scoring function

$$
f(P, Q, R ; P)=S\left(S_{1}, S_{2}, S_{3}\right) \ln P,
$$

which gives

$$
e(P, Q, R)=S(p \ln P+q \ln Q+r \ln R),
$$

to be minimised when $P=p, Q=q, R=r$.

On differentiating with respect to $P$ and rearranging, we find

$$
\begin{aligned}
& \frac{p}{P}=\frac{1}{S}\left(\frac{\partial S}{\partial S_{1}}+2 P \frac{\partial S}{\partial S_{2}}+3 P^{2} \frac{\partial S}{\partial S_{3}}\right)(p \ln P+q \ln Q+r \ln R), \\
& \frac{q}{Q}=\frac{1}{S}\left(\frac{\partial S}{\partial S_{1}}+2 Q \frac{\partial S}{\partial S_{2}}+3 Q^{2} \frac{\partial S}{\partial S_{3}}\right)(p \ln P+q \ln Q+r \ln R), \\
& \frac{r}{R}=\frac{1}{S}\left(\frac{\partial S}{\partial S_{1}}+2 R \frac{\partial S}{\partial S_{2}}+3 R^{2} \frac{\partial S}{\partial S_{3}}\right)(p \ln P+q \ln Q+r \ln R),
\end{aligned}
$$

and these equations are to be identically satisfied by $P=p, Q=q, R=r$.

Simplifying these equations results in

$$
S[(p Q-q P) R(Q-R)-(q R-r Q) P(P-Q)]=3 \frac{\partial S}{\partial S_{3}}(p \ln P+q \ln Q+r \ln R) .
$$

But now this must be satisfied by $P=p, Q=q, R=r$, for all allowable $p, q$, $r$ and this implies that $\partial S / \partial S_{3}=0$. Back substitution now yields the further result $\partial S / \partial S_{2}=0$. Thus

$$
f(P, Q, R ; P)=S\left(S_{1}\right) \ln P=S(P+Q+R) \ln P
$$

and the minimisation criterion reduces to

$$
\frac{p}{P}=\frac{q}{Q}=\frac{r}{R}=\frac{S^{\prime}(P+Q+R)}{S(P+Q+R)}(p \ln P+q \ln Q+r \ln R),
$$

which cannot in general be satisfied by $(P, Q, R)=(p, q, r)$.

A similar analysis applies if we impose the condition $P+Q+R=1$ and apply Lagrange multiplier techniques. The details are here omitted. We are left with the case $f(P, Q, R ; P)=\ln P$.

\section{Non-logarithmic scoring functions}

Now consider the other component of the scoring function:

$$
f(P, Q, R ; P)=A\left(S_{1}, S_{2}, S_{3}\right)+P B\left(S_{1}, S_{2}, S_{3}\right)+P^{2} C\left(S_{1}, S_{2}, S_{3}\right) .
$$


This gives

$$
\begin{aligned}
e(P, Q, R)= & (p+q+r) A\left(S_{1}, S_{2}, S_{3}\right)+(p P+q Q+r R) B\left(S_{1}, S_{2}, S_{3}\right) \\
& +\left(p P^{2}+q Q^{2}+r R^{2}\right) C\left(S_{1}, S_{2}, S_{3}\right)
\end{aligned}
$$

For convenience, write $s_{1}=p+q+r, s_{2}=p^{2}+q^{2}+r^{2}, s_{3}=p^{3}+q^{3}+r^{3}$. The condition that $e(P, Q, R)$ be extremised for the choice $(P, Q, R)=(p, q, r)$ may now be addressed.

The partial derivatives of $e(P, Q, R)$ may be formed and set equal to zero. These partial derivatives will be mutually independent, as we do not yet impose the condition $P+Q+R=1$.

We have

$$
\begin{aligned}
\frac{\partial e}{\partial P}= & (p+q+r) \frac{\partial A}{\partial P}+(p P+q Q+r R) \frac{\partial B}{\partial P} \\
& +\left(p P^{2}+q Q^{2}+r R^{2}\right) \frac{\partial C}{\partial P}+p(B+2 P C) \\
= & (p+q+r)\left(\frac{\partial A}{\partial S_{1}}+2 P \frac{\partial A}{\partial S_{2}}+3 P^{2} \frac{\partial A}{\partial S_{3}}\right) \\
& +(p P+q Q+r R)\left(\frac{\partial B}{\partial S_{1}}+2 P \frac{\partial B}{\partial S_{2}}+3 P^{2} \frac{\partial B}{\partial S_{3}}\right) \\
& +\left(p P^{2}+q Q^{2}+r R^{2}\right)\left(\frac{\partial C}{\partial S_{1}}+2 P \frac{\partial C}{\partial S_{2}}+3 P^{2} \frac{\partial C}{\partial S_{3}}\right)+p(B+2 P C)
\end{aligned}
$$

which must be identically equal to zero if we put $(P, Q, R)=(p, q, r)$ and recall that $p+q+r=1$.

If we make the substitution $(P, Q, R)=(p, q, r)$, we find

$$
\begin{aligned}
\left.\frac{\partial e}{\partial P}\right|_{(P, Q, R)=(p, q, r)}= & \left(s_{1} \frac{\partial A}{\partial s_{1}}+s_{2} \frac{\partial A}{\partial s_{2}}+s_{3} \frac{\partial A}{\partial s_{3}}\right)+2 p\left(s_{1} \frac{\partial B}{\partial s_{1}}+s_{2} \frac{\partial B}{\partial s_{2}}+s_{3} \frac{\partial B}{\partial s_{3}}\right) \\
& +3 p^{2}\left(s_{1} \frac{\partial C}{\partial s_{1}}+s_{2} \frac{\partial C}{\partial s_{2}}+s_{3} \frac{\partial C}{\partial s_{3}}\right),
\end{aligned}
$$

where now $A=A\left(s_{1}, s_{2}, s_{3}\right)$, and so on.

Because this expression is to be identically zero, the individual terms are to be zero, but subject to the condition $s_{1}=p+q+r=1$. We thus have

$$
\begin{aligned}
& s_{1} \frac{\partial A}{\partial s_{1}}+s_{2} \frac{\partial B}{\partial s_{1}}+s_{3} \frac{\partial C}{\partial s_{1}}=\left(s_{1}-1\right) \alpha\left(s_{1}, s_{2}, s_{3}\right), \\
& s_{1} \frac{\partial A}{\partial s_{2}}+s_{2} \frac{\partial B}{\partial s_{2}}+s_{3} \frac{\partial C}{\partial s_{2}}=\left(s_{1}-1\right) \beta\left(s_{1}, s_{2}, s_{3}\right)-B / 2,
\end{aligned}
$$




$$
s_{1} \frac{\partial A}{\partial s_{3}}+s_{2} \frac{\partial B}{\partial s_{3}}+s_{3} \frac{\partial C}{\partial s_{3}}=\left(s_{1}-1\right) \gamma\left(s_{1}, s_{2}, s_{3}\right)-2 C / 3,
$$

where $\alpha, \beta, \gamma$ are (at present) arbitrary functions of the stipulated arguments.

In order to proceed further, write

$$
\phi=s_{1} A+s_{2} B+s_{3} C .
$$

The equations then become

(10) $\frac{\partial \phi}{\partial s_{1}}=A+\left(s_{1}-1\right) \alpha, \quad \frac{\partial \phi}{\partial s_{2}}=\frac{B}{2}+\left(s_{1}-1\right) \beta, \quad \frac{\partial \phi}{\partial s_{3}}=\frac{C}{3}+\left(s_{1}-1\right) \gamma$,

Multiply the first of these equations by $s_{1}$, the second by $2 s_{2}$, the third by $3 s_{3}$ and add. This yields

$$
s_{1} \frac{\partial \phi}{\partial s_{1}}+2 s_{2} \frac{\partial \phi}{\partial s_{2}}+3 s_{3} \frac{\partial \phi}{\partial s_{3}}=\phi+\left(s_{1}-1\right) \theta\left(s_{1}, s_{2}, s_{3}\right)
$$

where

$$
\theta=s_{1} \alpha+2 s_{2} \beta+3 s_{3} \gamma
$$

\section{Some simple cases}

Before turning to more general cases, consider the special case in which $\phi$ is independent of $s_{1}$. In that case, we must have $\alpha=\beta=\gamma=\theta=0$ so that (10) become:

$$
\partial \phi / \partial s_{2}=B / 2, \quad \partial \phi / \partial s_{3}=C / 3
$$

and (11) becomes

$$
2 s_{2} \frac{\partial \phi}{\partial s_{2}}+3 s_{3} \frac{\partial \phi}{\partial s_{3}}=\phi
$$

This has the general solution (see $[4,867-870]$ )

$$
\phi=\sqrt{s_{2}} \psi\left(s_{3}^{2} / s_{2}^{3}\right) .
$$

In order to complete the analysis for these cases, all that is required is to revert to the capital letters and to apply (13).

In a particularly simple case, choose $\psi=1$, and so reach the 'spherical' scoring function [8], which is characterised by $A=C=0, B=1 / \sqrt{S_{2}}$ and is the simplest of an infinite set of possibilities. Another special case $\psi=\left(S_{3}^{2} / S_{2}^{3}\right)^{2 / 3}$ gives a slightly more complicated scoring function characterised by $A=B=0, C=\left(S_{3}\right)^{-2 / 3}$. 


\section{Solution of the general partial differential equation}

Apart from the degenerate case just examined, (11) is a linear partial differential equation whose general solution [4, pages $867-870$ ] is

$$
\phi=\phi\left(s_{1}, \frac{s_{2}}{s_{1}^{2}}, \frac{s_{3}}{s_{1}^{3}}\right)=\phi\left(s_{1}, x, y\right) \quad \text { (say), }
$$

with the further condition

$$
\frac{\partial \phi}{\partial s_{1}}=\phi+\left(s_{1}-1\right) \theta\left(s_{1}, x, y\right)
$$

where the partial derivative is now taken keeping $x, y$ constant rather than as before with $s_{1}, s_{2}$ constant. It will also be noted that the dependence of $\theta$ on $s_{1}, s_{2}$ must be mediated as a dependence on $x, y$ for consistency in (16).

Equation (16) is a further linear partial differential equation and this may also be solved. The integrating factor is $s_{1}^{-2}$ and it yields

$$
\frac{\partial}{\partial s_{1}}\left(\frac{\phi}{s_{1}}\right)=\frac{s_{1}-1}{s_{1}^{2}} \theta \text {. }
$$

It will be convenient to write

$$
\theta=s_{1}^{2} \frac{\partial^{2}}{\partial s_{1}^{2}} \chi\left(s_{1}, x, y\right) .
$$

(A similar device was adopted in the earlier study [2].)

The general solution of (17) is

$$
\phi=s_{1}\left(s_{1}-1\right) \frac{\partial \chi}{\partial s_{1}}-s_{1} \chi+s_{1} \kappa(x, y),
$$

where $\kappa$ is arbitrary. However, the function $\kappa$ may be omitted as it may be absorbed into the $\chi$, that is to say we may replace $\chi-\kappa$ by the simpler $\chi$ and so reach

$$
\phi=s_{1}\left(s_{1}-1\right) \frac{\partial \chi}{\partial s_{1}}-s_{1} \chi
$$

as the solution of the equation, where for consistency we now require

$$
s_{1} \alpha+2 s_{2} \beta+3 s_{3} \gamma=s_{1}^{2} \frac{\partial^{2} \chi}{\partial s_{1}^{2}}
$$

following the various changes of notation.

Thus the scoring function depends on a function $\chi$, in its turn depending on $s_{1}$, $x, y$ and from which another function $\phi$ is constructed by means of (19). Once $\phi$ is determined, then $A, B, C$ may be found by means of (10) under the condition (20). [But note the differing conventions between (10) and (20). In the former case, it is the individual $s_{i}$ that are held constant during the differentiation; in the latter it is $x, y$.] 
It may be remarked that the functions discussed in Section 6 may be recovered by means of the choice $\chi=-\sqrt{x} \psi\left(y^{2} / x^{3}\right)$. Further discussion will be confined to a restricted but important subset of the possible functions that could be employed. That subset is the set of integral functions. Thus, although the analysis is on that account somewhat incomplete, a richness of possible scoring functions nonetheless emerges.

\section{Integral scoring functions}

Integral functions are those which may be expressed as sums of products of integral powers of the variables. In that case, where three variables are involved, and because $P, Q, R$ are the roots of a cubic equation whose coefficients are simple polynomials in $S_{1}, S_{2}, S_{3}$, then $P^{3}$ is quadratic in $P$ with coefficients functions of $S_{1}, S_{2}, S_{3}$, and thus this is also true for higher powers of $P$. Thus (6) holds immediately. This result is not given explicitly in Chrystal's text [1], but it readily follows from the discussion and the equations presented there (loc. cit.) In what follows, we restrict consideration to integral scoring functions.

Because scoring functions are additive, we may construct general cases from a smaller subset of special cases. The simplest cases are those in which $\chi$ is homogeneous in the variables $s_{1}, s_{2}, s_{3}$ so that $\chi=s_{1}^{m} \psi(x, y)$. Any other case can clearly be built up from a (finite or infinite) sum of such simpler cases. And the same may be said of the various possibilities for $\psi(x, y)$. Any such function may be written under our assumptions as a sum of functions of the form $\psi(x, y)=x^{\mu} y^{\nu}$.

Thus we may restrict consideration to the case

$$
\chi=s_{1}^{m} x^{\mu} y^{\nu},
$$

where for the function to be an integral one we require $m \geq 2 \mu+3 v$ and of course $\mu \geq 0, v \geq 0$. It will be convenient to write $m=\rho+2 \mu+3 v$. We may now discover from (18)

$$
\phi=(m-1) s_{1}^{m+1} x^{\mu} y^{\nu}-m s_{1}^{m} x^{\mu} y^{\nu}=(m-1) s_{1}^{\rho+1} s_{2}^{\mu} s_{3}^{\nu}-m s_{1}^{\rho} s_{2}^{\mu} s_{3}^{\nu} .
$$

We now have

$$
\begin{aligned}
\frac{\partial \phi}{\partial s_{1}}= & (2 \mu+3 \nu-1) s_{1}^{\rho} s_{2}^{\mu} s_{3}^{\nu}+(1-a) m \rho s_{1}^{\rho-1} s_{2}^{\mu} s_{3}^{v}\left(s_{1}-1\right) \\
& +a m \rho s_{1}^{\rho-1} s_{2}^{\mu} s_{3}^{\nu}\left(s_{1}-1\right) \\
\frac{\partial \phi}{\partial s_{2}}= & -\mu s_{1}^{\rho+1} s_{2}^{\mu-1} s_{3}^{\nu}+(1-b) \mu m\left(s_{1}-1\right) s_{1}^{\rho} s_{2}^{\mu-1} s_{3}^{\nu}+b \mu m\left(s_{1}-1\right) s_{1}^{\rho} s_{2}^{\mu-1} s_{3}^{v} \\
\frac{\partial \phi}{\partial s_{3}}= & -v s_{1}^{\rho+1} s_{2}^{\mu} s_{3}^{v-1}+(1-c) v m\left(s_{1}-1\right) s_{1}^{\rho} s_{2}^{\mu} s_{3}^{\nu-1}+c \nu m\left(s_{1}-1\right) s_{1}^{\rho} s_{2}^{\mu} s_{3}^{v-1}
\end{aligned}
$$


where we have taken the partial derivatives with respect to each of the three $s_{i}$ keeping the other two constant.

In (21), the new symbols $a, b, c$ represent real numbers and are (so far) otherwise arbitrary. The apparently clumsy form is chosen for comparison with the earlier equations (9). It gives, when that comparison is made,

$$
A=(2 \mu+3 v-1) s_{1}^{\rho} s_{2}^{\mu} s_{3}^{\nu}+(1-a) m \rho s_{1}^{\rho-1} s_{2}^{\mu} s_{3}^{\nu}\left(s_{1}-1\right),
$$

$$
\begin{gathered}
B / 2=-\mu s_{1}^{\rho+1} s_{2}^{\mu-1} s_{3}^{\nu}+(1-b) \mu m s_{1}^{\rho} s_{2}^{\mu-1} s_{3}^{\nu}\left(s_{1}-1\right), \\
C / 3=-\nu s_{1}^{\rho+1} s_{2}^{\mu} s_{3}^{\nu-1}+(1-c) \nu m s_{1}^{\rho} s_{2}^{\mu} s_{3}^{\nu-1}\left(s_{1}-1\right), \\
\alpha=a m \rho s_{1}^{\rho-1} s_{2}^{\mu} s_{3}^{\nu}, \quad \beta=b \mu m\left(s_{1}-1\right) s_{1}^{\rho} s_{2}^{\mu-1} s_{3}^{\nu}, \quad \gamma=\operatorname{c\nu m}\left(s_{1}-1\right) s_{1}^{\rho} s_{2}^{\mu} s_{3}^{\nu-1} .
\end{gathered}
$$

It still remains to satisfy (20). To do this, equate the two values of $\theta$ given by (12) and (16). This produces the consistency requirement

$$
(a-1) \rho+2(b-1) \mu+3(c-1) v+1=0 .
$$

The final step in the analysis is to revert to the capital letters relevant to the prescription of the loss function $f(P, Q, R ; P)$. The final result thus has (7) with $A, B, C$ chosen as follows:

$$
\begin{aligned}
& A=(2 \mu+3 \nu-1) S_{1}^{\rho} S_{2}^{\mu} S_{3}^{\nu}+(1-a) m \rho S_{1}^{\rho-1} S_{2}^{\mu} S_{3}^{\nu}\left(S_{1}-1\right), \\
& B=-2 \mu S_{1}^{\rho+1} S_{2}^{\mu-1} S_{3}^{\nu}+2(1-b) \mu m S_{1}^{\rho} S_{2}^{\mu-1} S_{3}^{\nu}\left(S_{1}-1\right), \\
& C=-3 v S_{1}^{\rho+1} S_{2}^{\mu} S_{3}^{\nu-1}+3(1-c) v m S_{1}^{\rho} S_{2}^{\mu} S_{3}^{\nu-1}\left(S_{1}-1\right) .
\end{aligned}
$$

Under the constraint (23) this gives a fivefold infinity of candidate scoring functions, all of which satisfy the necessary condition for $e(P, Q, R)$ to be extremised by the choice $(P, Q, R)=(p, q, r)$.

Equations (22) may however be simplified by noting that now that all of the differentiations have been effected, it is safe to use the further information that $s_{1}=1$. If we insert this into (22) and then revert to capital letters, we reach a particularly simple set of solutions, in which (23) is irrelevant and $\rho$ is no longer present. These are:

$$
A=(2 \mu+3 v-1) S_{2}^{\mu} S_{3}^{\nu}, \quad B=-2 \mu S_{2}^{\mu-1} S_{3}^{\nu}, \quad C=-3 \nu S_{2}^{\mu} S_{3}^{\nu-1} .
$$

This is a doubly infinite family of possible scoring functions, which will be shown to be precisely those that arise if we stipulate in advance that $S_{1}=1$. But first, we turn to some illustrative special cases.

\section{Illustrative cases}

First confine attention to the simple equations (25). Set $\mu=1, v=0$, which gives $A=S_{2}, B=-2, C=0$, and this is precisely de Finetti's quadratic function (2) apart 
from the additive constant. We may also derive this case from (24) under the further conditions $\rho=0, b=1 / 2$, when

$$
\phi=s_{1} s_{2}-2 s_{2}, \quad \theta=2 s_{1}=2 s_{1}^{2} x, \quad \chi=s_{1}^{2} x .
$$

Another simple case to consider is $\mu=0, \nu=1$, which yields

$$
f(P, Q, R ; P)=2\left(P^{3}+Q^{3}+R^{3}\right)-3 P^{2} .
$$

And this may also be reached from (24) with the further conditions $\rho=0, c=2 / 3$. Similarly if $\mu=2, v=0$, we recover

$$
f(P, Q, R ; P)=3\left(P^{2}+Q^{2}+R^{2}\right)-4 P\left(P^{2}+Q^{2}+R^{2}\right),
$$

which can also be reached from (24) with $\rho=0, b=3 / 4$.

Another simple case is given by $\mu=\nu=1$ and this yields

$$
\begin{aligned}
f(P, Q, R ; P)= & 4\left(P^{2}+Q^{2}+R^{2}\right)\left(P^{3}+Q^{3}+R^{3}\right) \\
& -2 P\left(P^{3}+Q^{3}+R^{3}\right)-3 P^{2}\left(P^{2}+Q^{2}+R^{2}\right)
\end{aligned}
$$

which also may be reached from (24); the relevant parameter values are $\rho=0$, $b=3 / 5, c=14 / 15$.

All the above cases may be derived directly from the simplified equations (25) rather than from the full equations (24). The very simplest of the other cases that can be constructed from those more general solutions (apart from the trivial $\mu=\nu=0$ ) is $\rho=\mu=1, \nu=0$. This yields

$$
\begin{aligned}
f(P, Q, R ; P)= & (P+Q+R)\left(P^{2}+Q^{2}+R^{2}\right) \\
& +3(1-a)\left(P^{2}+Q^{2}+R^{2}\right)(P+Q+R-1) \\
& +P\left[2(P+Q+R)^{2}+3 a(P+Q+R)(P+Q+R-1)\right]
\end{aligned}
$$

and even in the simplest subcase ( $a=0$ ), detailed analysis is daunting. However it is possible in this instance, as a check, to find that the derivatives of $e(P, Q, R)$ indeed vanish at $(p, q, r)$, although I omit the details.

Now consider an apparently much more complicated case, whose motivation will become apparent.

Set

$$
\begin{aligned}
& A=\left[4 S_{1} S_{3}-3 S_{1}^{2} S_{2}+3 S_{2}^{2} / 2+S_{1}^{4} / 3\right]-4\left[S_{1}^{3} / 6-S_{1} S_{2} / 2+S_{3} / 3\right], \\
& B=2\left[S_{1}^{2}-S_{2}\right], \quad C=-4 S_{1},
\end{aligned}
$$

where setting $S_{1}=1$ results in a linear combination of cases discussed above together with some additive constants. The interest in this case resides in the identity

$$
f(P, Q, R ; P)=A+B P+C P^{2}=3\left(P^{4}+Q^{4}+R^{4}\right)-4 P^{3},
$$


where $A, B, C$ are defined by (26). That the right-hand side of (27) furnishes a suitable scoring function is readily checked. It lies as the third in a one-parameter family of possible scoring functions

$$
f(P, Q, R ; P)=(k-1)\left(P^{k}+Q^{k}+R^{k}\right)-k P^{k-1}
$$

whose earlier members are given above.

This last example may be generated from the function

$$
\chi\left(s_{1}, x, y\right)=-\frac{1}{6} s_{1}^{4}\left(1-6 x+8 y+3 x^{2}\right) .
$$

The details are left to the reader.

\section{Constrained extrema}

If, at the outset, we impose the condition $S_{1}=1$, that is to say we force the tipster to nominate probabilities for the three outcomes, then equations (9) are replaced by the simpler:

$$
\frac{\partial \phi}{\partial s_{1}}=A+\lambda, \quad \frac{\partial \phi}{\partial s_{2}}=\frac{B}{2}, \quad \frac{\partial \phi}{\partial s_{3}}=\frac{C}{3},
$$

where $\lambda$ is a Lagrange multiplier. Equation (11) is now replaced by

$$
s_{1} \frac{\partial \phi}{\partial s_{1}}+2 s_{2} \frac{\partial \phi}{\partial s_{2}}+3 s_{3} \frac{\partial \phi}{\partial s_{3}}=\phi+\lambda s_{1}
$$

and the solution of this partial differentiation is

$$
\phi=\lambda+s_{1} \psi(x, y) .
$$

As in Section 9 above, set $\psi(x, y)=x^{\mu} y^{\nu}$ to discover basic solutions. Equations (28) now yield:

$$
A=(1-2 \mu-3 v), \quad B=2 \mu x^{\mu-1} y^{\nu}, \quad C=3 v x^{\mu} y^{\nu-1}
$$

which, apart from sign, yields (25).

The requirement $m \geq 2 \mu+3 v$ (valid in the earlier analysis, but here not satisfied, as $m=1$ ) is no longer applicable, as the $s_{1}$ in the denominators is constrained to be 1 . It should also be remarked that this subset of solutions may also be reached without imposing the condition $S_{1}=1$. 


\section{Acknowledgments}

I thank David Dowe for bringing this problem to my attention and for introducing me to the work of Winkler, and I am indebted to an anonymous referee for further references.

\section{References}

[1] G. Chrystal, Algebra: an elementary textbook, 7th edition (Chelsea, New York, 1964).

[2] M. A. B. Deakin, 'Scoring functions for football-tipping and other such contests', Gaz. Aust. Math. Soc. 26 (1999), 15-18.

[3] B. de Finetti, Theory of probability, Vol. I (Wiley, London, 1974), Ch. 5.

[4] K. Rektorys et al, Survey of applicable mathematics, English edition (Iliffe, London, 1969).

[5] L. J. Savage, 'Elicitation of personal probabilities and expectations', J. Amer. Statist. Assoc. 66 (1971), 783-801.

[6] M. J. Schervish, 'A general method for comparing probability assessors', Ann. Statist. 17 (1989), 1856-1879.

[7] R. L. Winkler, 'Scoring rules and the evaluation of probability assessors', J. Amer. Statist. Assoc. 64 (1969), 1073-1078.

[8] —- 'Judgments under uncertainty', in: Encyclopaedia Statist. Sci., ('Kotz-Johnson') Vol. 4 (Wiley, New York, 1983) pp. 332-336.

[9] _..., 'Scoring rules and the evaluation of probabilities', Test 5 (1996), 1-60.

Department of Mathematics and Statistics

Monash University

Clayton Vic 3168

Australia

e-mail:michael.deakin@sci.monash.edu.au 